\title{
Trypanosomatid-Encoded Inhibitors of Peptidases: Unique Structural Features and Possible Roles as Virulence Factors
}

\author{
Ana Paula C.A. Lima ${ }^{* 1}$ and Jeremy C. Mottram ${ }^{2}$ \\ ${ }^{I}$ Instituto de Biofisica Carlos Chagas Filho, Centro de Ciencias da Saude, Bloco G, Universidade Federal do Rio de \\ Janeiro, Cidade Universitaria, Ilha do Fundao, Rio de Janeiro, RJ, 21949-900, Brazil \\ ${ }^{2}$ Wellcome Trust Centre for Molecular Parasitology and Division of Infection and Immunity, Faculty of Biomedical and \\ Life Sciences, University of Glasgow, Glasgow G12 8TA, UK
}

\begin{abstract}
Trypanosomatids rely on peptidases as potent virulence factors and were recently found to contain a unique set of natural peptidase inhibitors not found in higher eukaryotes or in yeast, but present in a limited number of bacteria. Chagasin, identified in Trypanosoma cruzi, is a tight-binding, high affinity inhibitor of papain-like cysteine peptidases that has an Ig-like fold and inactivates target enzymes through a limited number of contacts mediated by a few conserved residues on three exposed loops. Chagasin homologues in other protozoa and bacteria are mostly single genes named ICPs, and together with chagasin compose family I42 at MEROPS, the peptidase and peptidase inhibitors database [http://merops.sanger.ac.uk/]. The biological function of chagasin/ICPs seems to vary depending on the organism, but generally, the current studies point to a role in controlling the activity of endogenous parasite CPs, influencing parasite differentiation, virulence and different aspects of the host response to infection. More recently, natural inhibitors of serine peptidases that share similarity to bacterial ecotins were identified in trypanosomatids and named ISPs. Ecotins are specific to trypsin-fold serine peptidases, enzymes which are not present in trypanosomatids. ISPs are limited to Trypanosomes and Leishmania and to date, only ISP2 proven to have an inhibitory function. In Leishmania, ISP2 seems to control the activity of host SPs at the initial stages of infection in order to ensure subsequent parasite survival in macrophages. The main aspects of chagasin/ICP biochemistry, structure and biological function and the recent findings on ISPs will be covered in this review.
\end{abstract}

Keywords: Trypanosoma, trypanosomatid-encoded inhibitors of peptidases, structural features, virulence factor, cysteine peptidase, serine peptidase.

\section{INTRODUCTION}

Peptidase inhibitors are widely distributed in nature and are an important mechanism of control of proteolytic activity. Up to 2010, there are more than 19977 natural peptidase inhibitors described in the MEROPS database, distributed in 67 families and the three dimensional structures of 160 have been elucidated. In mammals and plants a great variety of proteinaceous inhibitors, produced in a cell or tissue specific manner, help to control the degree of proteolytic activity during normal physiology processes such as hormone processing, coagulation, antigen-presentation and cellular remodeling and changes in their expression levels or modes of action have been associated with pathologies such as cancer, inflammation, neurodegenerative disorders and infection [1, 2]. Peptidase inhibitors generally exert their function via specific and high affinity interactions with their target peptidases, ultimately blocking the access of potential substrates to the enzymes' active sites. Most of the studies regarding the biology of natural peptidase inhibitors were carried out in multi-cellular organisms, or their cells, where the need for

*Address correspondence to this author at the Instituto de Biofisica Carlos Chagas Filho, Centro de Ciencias da Saude, Bloco G, Universidade Federal do Rio de Janeiro, Cidade Universitaria, Ilha do Fundao, Rio de Janeiro, RJ, 21949-900, Brazil; Tel: (+55) 21-2209-6591; Fax: (+55) 21-2280-8193; E-mail: anapaula@biof.ufrj.br tight control of proteolysis arose to prevent untimely cell death or tissue damage. In contrast, the biological roles of natural peptidase inhibitors in unicellular organisms remain less clear and might reflect adaptions in their relationships with peptidase-rich hosts or with the environment.

In the past years, solid evidence has shown that pathogenic trypanosomatids rely largely on peptidases as virulence factors $[3,4]$ and these molecules have become attractive drug targets. In the genomes of Trypanosoma cruzi (the etiological agent of Chagas Disease), Leishmania major (responsible for cutaneous leishmaniasis) and Trypanosoma brucei (causative agent of sleeping sickness), more than 150 genes encoding peptidases distributed in all classes have been identified. Members of the papain-family of cysteine peptidases (CPs) were established as promising targets for chemotherapy, and their functions have been associated with parasite growth, differentiation and infection [3].However, the existence of an endogenous control for the activity of peptidases in these organisms remained elusive for a long time. In the early 90 `s, a single biochemical study pointed to the presence of heat-resistant activity in extracts of Leishmania, that was capable of inhibiting papain [5] but it was not until 2001 that a natural inhibitor of papain-like peptidases, chagasin, was characterized in T. cruzi [6]. Presently, the chagasin-family comprises several members, identified in protozoa and bacteria, and a growing number of studies in 
pathogenic protozoa identify these molecules as potent regulators of cysteine peptidases of both the parasite and the host, contributing to the outcome of infection [7-13].

\section{STRUCTURAL AND BIOCHEMICAL FEATURES}

\section{Chagasin Family (ICPs)}

Chagasin is a 110 amino acid polypeptide first identified in lysates of $T$. cruzi epimastigotes by biochemical assays indicating the presence of a heat-resistant, low molecular mass molecule capable of high affinity inhibition of several papain-like cysteine peptidases [6]. Classic biochemical approaches led to the identification of a protein with a novel $\mathrm{N}$ terminal sequence, which matched a parallel genetic screen of $T$. cruzi expression libraries using papain as bait. These studies revealed the presence of a single gene in $T$. cruzi, bearing a unique predicted amino-acid sequence that showed no significant similarity to known peptidase inhibitors at the time [6]. Natural and recombinant chagasin share physicalchemical properties with cystatins, and are tight, reversible, high affinity inhibitors of papain-like enzymes, forming 1:1 complexes. It soon became clear that other protozoa and bacteria carried genes sharing similarity $(<25 \%)$ with chagasin. This was limited to small regions, mainly a short stretch of 5 residues close to the N-terminal region (NPTTG) and a tripeptidade close to the C-terminus (RPW), considered the motifs of chagasin-like inhibitors [7]. The recombinant proteins derived from such genes of L. mexicana, T. brucei and Pseudomonas aeruginosa were high affinity inhibitors of papain, and were named ICP (Inhibitors of Cysteine Peptidases) [8], being grouped with chagasin in a new family of cysteine peptidase inhibitors, classified as family IX in MEROPS. Its limited distribution in protozoa and bacteria led to the proposal of horizontal gene transfer, possibly during the co-existence of the parasite and bacteria in the gut of the insect vector [8]. Later, the chagasin-like genes of Plasmodium and Entamoeba [10-12] were also proven to be functional inhibitors, adding to the growing list of the unique chagasin family. Early in silico studies suggested that the evolutionary conserved residues of chagasin were exposed in loops to one side of the molecule, forming interactions with the target peptidases [7]. The solution structures of chagasin and $L$. mexicana ICP revealed that these proteins exhibit an Ig-like fold, a feature which had not been found before in other peptidase inhibitors, and confirmed that the conserved residues were arranged in loops to one side of the molecule, L2, L4 and L6 [14, 15]. These key three loops contain respectively: (i) the NPTTG motif, (ii) a conserved region comprised by two hydrophobic residues followed by GXGG and (iii) the RPW/F motif. Interestingly, both studies revealed that L4 is highly mobile in solution, a feature most probably attributable to the presence of multiple glycine residues. Analyses of chemical shift perturbations of chagasin during contact with cruzipain (the papain-like protease of T. cruzi) [16] suggested that L2 and L6 would make contact with the enzyme [14]. Later, computational analyses of docking of the chagasin crystal to the crystal of cruzain [17], the recombinant form of cruzipain truncated at the $\mathrm{C}$ terminal extension, and the determination of the complex structures of chagasin-falcipain2 and chagasin-cathepsin L, confirmed that the conserved residues in L2 are inserted into the peptidases' active sites [18, 19]. The main conclusions derived from the earlier structural data suggested that the interactions between residues Thr-31 and Thr-32 (L2) of chagasin with the catalytic triad of the peptidases through water mediated hydrogen bonds and hydrophobic interactions of residues in L6 with the peptidase would ultimately contribute to high affinity inhibition. In Reis et al., joint studies between my group and the group of Mottram evaluated the relative contribution of conserved residues in chagasin to inhibitor function by mutagenesis, revealing a more complex scenario [20]. We found that the structural basis for high affinity inhibition of papain-like enzymes by chagasin varies according to the target enzyme [20]. Strikingly, substitutions in Thr-31 or Thr-32 (L2) of chagasin affected dramatically the affinity for cruzipain, the main parasite cysteine protease, while it had a negligible effect on the inhibition of cathepsin L, a host derived peptidase. In contrast, a mutation of the hydrophobic Trp-93 residue (L6), lowered the affinity of chagasin for cathepsin L by 100 -fold, with minimal effect on the affinity for cruzipain [20]. Furthermore, we found that shortening of L2 by removal of Thr-31 and Thr-32 caused a substantial reduction in the inhibitory potential of chagasin, regardless of the target peptidase tested.

The determination of the X-ray structures of additional chagasin: peptidase complexes revealed a clearer picture of the mode of interaction of this novel inhibitor [21, 22]. The main conclusions drawn from those studies are that: (i) the overall conformation of chagasin in complex with enzymes is very similar to that of free chagasin; and (ii) it maintains a very conserved mode of interaction with target peptidases, with all three loops located in the catalytic groove, the tip of L2 inserted directly into the active site, and loops L4 and L6 embracing the enzyme from each side. The nature of interactions with L6 is mainly hydrophobic stacking of the conserved Trp-93 residue with a hydrophobic patch conserved in most $\mathrm{C} 1$ peptidases and serving as an important anchor. These studies elegantly showed that chagasin approaches the target peptidases at different angles, adjusting to a final rigid and similar conformation in all the complexes, even though enzyme-specific interactions can be mapped. A common feature in all the complex structures available is that while the interactions with L2 are somewhat limited, despite its insertion inside the catalytic groove, the more extensive and very strong interactions with loops L4 and L6 likely enable a tight grip of the enzymes, conferring chagasin its property as a high affinity inhibitor. Can we extrapolate these conclusions to the interaction between chagasin and its endogenous target, cruzipain? Although it can be predicted with reasonable confidence that the overall mode of binding will be conserved, our mutagenesis data points to an important contribution of residues in $\mathrm{L} 2$ for the inhibition of cruzipain. The determination of the structure of the chagasin: cruzipain complex will allow us to define additional and/or specific interactions that could ultimately help in the confection of more potent and selective drugs to the parasite's peptidase.

Chagasin makes very few direct contacts with the active sites of the peptidases it inhibits, utilizing a few conserved residues of the target enzymes to become a broad-reactive inhibitor with comparable affinity for $\mathrm{C} 1$ peptidases. A demonstration of its potential as a broad inhibitor is the inactivation of Cathepsin B, albeit with lower affinity as compared 
to the inhibition of cathepsin L, papain and cruzipain [21]. Cathepsin B is characterized by the presence of an occluding loop that blocks part of the active site, and confers its exopeptidase activity [23]. At first, it was hard to imagine how chagasin could overcome this obstacle in order to cause enzyme inhibition. It was then shown that chagasin can displace the occluding loop of cathepsin B, pushing it out of the active site through interactions with L6, which results in an enlargement of the enzyme-inhibitor interface [21]. Importantly, the comparison of the chagasin: papain complex with the stefin B (a type 1 cystatin):papain complex led to the striking conclusion that although cystatins and chagasin have a completely different fold, the epitopes they present to the enzymes are arranged very similarly [22]. In this sense, the L4-L2-L6 wedge of chagasin overlaps with the wedge of cystatin, formed by the N-terminal segment and the two $\alpha$ hairpin loops, a similarity that does not extend to the rest of the molecules. This is a good example of how convergent evolution resulted in two structurally different inhibitors displaying similar tight-binding, high affinity inhibition of $\mathrm{C} 1$ family peptidases.

\section{Inhibitors of Serine Peptidases (ISPs)}

The discovery of chagasin prompted the search for additional natural peptidase inhibitors in the TriTryp genomes. Interestingly, the only other putative genes for natural peptidase inhibitors encountered showed similarity to a natural inhibitor of serine peptidases limited to a few genera of bacteria, ecotin [24]. Ecotin was first described as a periplasmic low molecular mass protein in E. coli, and homologues are distributed over 15 genera of bacteria that, with two exceptions, are found in association with mammals [25, Comprehensive Microbial Resource: http://cmr.jcvi.org/cgibin/CMR/CmrHomePage.cgi]. Ecotins function as dimeric inhibitors and are highly selective for trypsin-fold serine peptidases belonging to the S1A family. A vast number of studies have evaluated the structural features of ecotin, revealing that each monomer contains a primary binding site that interacts directly with the enzyme`s active site while two additional secondary binding sites are formed as surface loops in the dimers, and are important for defining inhibitor affinity for different target peptidases [26-30]. The dimer is a tight-binding high affinity inhibitor that binds two enzyme molecules, creating a stable tetramer. A single study of the possible biological role of ecotin points to a function in protecting $E$. coli from the deleterious effect of neutrophil elastase (NE), secreted by neutrophils [31].

The three ecotin-like genes identified in L. major were named $I S P 1, I S P 2$ and $I S P 3$ (Inhibitors of Serine Peptidases) and are also present in other Leishmania species [24]. While $I S P 3$ is exclusive to Leishmania, ISP1 and ISP2 are present in $T$. brucei while only ISP2 was found in T. cruzi. ISP 3 is predicted to encode a protein formed with an $\mathrm{N}$-terminal ecotin-like domain followed by a unique $\mathrm{C}$-terminal extension with no similarity to known domains however the expression of $I S P 3$ cannot be detected in L. major. Importantly, trypanosomatids apparently lack genes encoding S1A peptidases, suggesting that the targets of ISPs are serine peptidases from their hosts. Differently from ICPs, that are present in a number of protozoa (Table 1), ISPs are found only in trypanosomes and Leishmania and show a significant de- gree of similarity to each other and to ecotin $(\sim 30 \%)$. As expected, the conserved domains are concentrated in the primary and secondary binding sites, but differently from ecotins, there is no predicted signal peptide in ISPs. The $L$. major ISP2 gene encodes a heat-resistant, $\sim 16 \mathrm{KDa}$ functional inhibitor that inactivates trypsin, chymotrypsin and neutrophil elastase, albeit showing 100-fold less affinity for those peptidases than ecotin does [24]. Likewise, T. cruzi ISP2 is a functional inhibitor, presenting properties similar to those of L. major ISP2 (Abreu MF, Reis FCG, Mottram JC, Lima APCA, unpublished). The inhibitory potential of ISP1 is not clear, but our initial studies suggest that it inactivates neutrophil elastase, while its inhibitory activity against trypsin and chymotrypsin is less evident (Lima APCA, Mottram $\mathrm{JC}$, unpublished). The three dimensional structure of the ISPs is unknown and it is still to be determined whether they function as homodimers. However, based on the initial biochemical characterization of the ISPs and on substitutions encountered in their predicted primary binding sites, in comparison to ecotin, we can anticipate that the resolution of the $I S P$ : peptidase complex structures will reveal particular features of the mode of interaction between the parasites' inhibitors and target enzymes.

\section{DISTRIBUTION, EXPRESSION AND ROLES IN PARASITE BIOLOGY AND INFECTION}

\section{Chagasin (ICPs)}

Chagasin is expressed in all life stages of $T$. cruzi, being more abundant in the mammalian infective trypomastigote stage. Immunocytochemistry analyses showed a complex sub-cellular distribution, being evident at the surface and flagellar pocket of tissue culture trypomastigotes and trypomastigote-amastigote intermediate forms, while in epimastigotes it is mainly intracellular [6]. In later studies, chagasin was detected in the Golgi apparatus, in reservosomes (a late endocytic compartment of T. cruzi), and in cytoplasmic vesicles containing cruzipain or devoid of the enzyme [32]. The presence of chagasin in reservosomes was recently confirmed in a proteomic study [33]. Reservosomes are the main deposits of active cruzipain [34], and the presence of chagasin therein strongly suggested that inhibitor: peptidase complexes could be naturally present in $T$. cruzi. Indeed, we showed the existence of such complexes in epimastigotes, both intracellularly and in the medium [32]. Considering that cruzipain zymogens are activated in the Golgi, it is possible that association with chagasin occurs early in the secretory pathway and is further accomplished when vesicles transporting free chagasin fuse with reservosomes containing active enzyme. Even though some mature cruzipain seems to be kept inactive in tight complexes with chagasin, the peptidase levels are approximately in 50-fold excess in relation to chagasin, demonstrating that the vast majority of cruzipain is free. The low level expression of chagasin enabled us to use chagasin-overexpressing parasites as a genetic tool to address inhibitor function. In $T$. cruzi lines expressing 5 -fold more chagasin than wild type, there was an $85 \%$ reduction in the amounts of active cruzipain, and significant effects on metacyclogenesis and in parasite resistance to the deleterious effect of synthetic irreversible inhibitors to cruzipain [32]. Moreover, chagasin-overexpressing trypomastigotes were less infective in vitro, and this was overcome by the addition 
Table 1. Peptidase Inhibitors of Protozoa. The table summarizes the main characteristics of the cysteine peptidase inhibitors and serine peptidase inhibitors described in protozoa. The sub-cellular distribution is described for the different life cycle stages (letters in brackets), according to the abbreviations found in the "expression" column. The three-dimensional structures of chagasin complexed with cysteine peptidase are listed in the "structure" column.

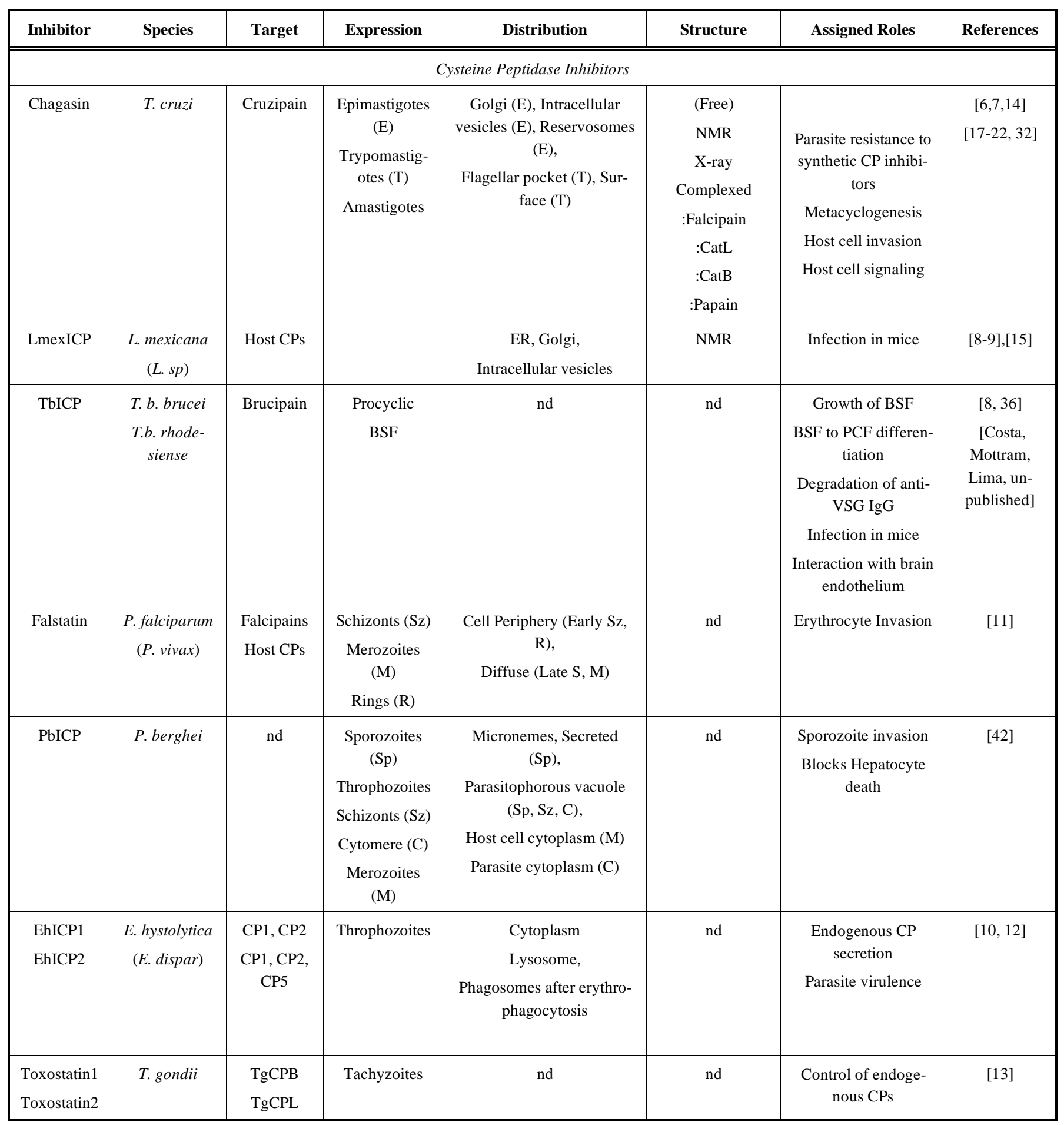


(Table 1). Contd.....

\begin{tabular}{|c|c|c|c|c|c|c|c|}
\hline Inhibitor & Species & Target & Expression & Distribution & Structure & Assigned Roles & References \\
\hline \multicolumn{8}{|c|}{ Serine Peptidase Inhibitors } \\
\hline $\begin{array}{l}\text { ISP1 } \\
\text { ISP2 } \\
\\
\text { ISP3 }\end{array}$ & L. major & $\begin{array}{c}\text { nd } \\
\text { Host SPs } \\
\text { nd }\end{array}$ & $\begin{array}{c}\text { Promastigotes, } \\
\text { Metacyclics } \\
\text { Promastigotes, } \\
\text { Metacyclics, } \\
\text { Amastigotes } \\
\text { Not detected }\end{array}$ & nd & nd & $\begin{array}{l}\text { Macrophage inva- } \\
\text { sion, } \\
\text { Parasite intracellular } \\
\text { survival }\end{array}$ & {$[24]$} \\
\hline $\begin{array}{l}\text { ISP1 } \\
\text { ISP2 }\end{array}$ & $\begin{array}{l}\text { T. b. brucei } \\
\text { T.b. rhode- } \\
\quad \text { siense }\end{array}$ & nd & $\begin{array}{c}\text { Procyclic } \\
\text { BSF }\end{array}$ & nd & nd & nd & $\begin{array}{c}\text { Genedb } \\
\text { Tb927.5.173 } \\
0 \\
\text { Tb927.5.188 } \\
0\end{array}$ \\
\hline ISP2 & T. cruzi & nd & $\begin{array}{c}\text { Epimastigotes } \\
\text { Trypomastigotes } \\
\text { Amastigotes }\end{array}$ & nd & nd & nd & $\begin{array}{c}\text { Genedb } \\
\text { Tc00.104705 } \\
3508533.40\end{array}$ \\
\hline
\end{tabular}

Nd, not determined; CP, cysteine peptidases (C1 family); CatL, cathepsin L; CatB, cathepsin B; ER, endoplasmic reticulum; BSF, bloodstream form, SP, serine peptidase (S1A family).

of exogenous cruzipain [32]. These data support the notion that subtle variations in the levels of chagasin expression affected important biological parameters associated with $\mathrm{CP}$ activity, establishing that chagasin plays a role in regulating endogenous CPs in T. cruzi.

A more detailed characterization of trypomastigotes overexpressing chagasin revealed an even greater impact on the content of active endogenous cruzipain, with increased levels of secreted enzyme: inhibitor complexes (Santos CC, Lima APCA, unpublished). The nature of the signaling pathways triggered by the parasite overexpressing chagasin upon invasion of the host cell were altered, suggesting that chagasin may play a further role in controlling the ways through which the parasite interacts with the host. It has been described that cruzipain inactivated by pharmacological inhibitors protects mouse cardyomyocytes from apoptosis in culture, mimicking the protective effect caused by parasite infection [35]. Recently, a thorough study of these properties revealed that active cruzipain was unable to protect cardiac cells from apoptosis, but this was fully reversed upon complexation with chagasin (Aoki MP, Lima APCA, Gea S, unpublished). The protective effect of inactive cruzipain correlated with the production of cytokines by the cardiac cells, establishing an association between cruzipain inactivated by chagasin, innate responses and potential protection of tissue damage. These observations suggested that cruzipain: chagasin complexes secreted by the parasite might influence the degree of preservation of the heart during infection, contributing to the outcome of pathology.

In $T$. brucei, ICP seems to be expressed at much lower levels than its counterparts in Leishmania and T. cruzi, and its sub-cellular location remains inconclusive [36]. Nevertheless, in the T. brucei bloodstream form (BSF) ICP seems to regulate the function of the endogenous CPs that plays a part in surface protein turnover, at least so in the removal of the variant surface glycoprotein (VSG) during differentiation and in the degradation of anti-VSG IgGs [36]. We found that $T$. brucei lines lacking ICP have increased levels of active CPs, leading to a more efficient intracellular degradation of antibodies to VSG, after endocytosis [36]. Moreover, the differentiation of $T$. brucei $\mathrm{BSF}$ to the procyclic form in vitro was accelerated in ICP-deficient lines, implicating CP activity in parasite differentiation [36]. ICP-deficient BSF were also more virulent to mice, reaching 10 -fold higher parasitemia in the blood and leading to premature death of the infected animals [36]. Based on these observations, we might predict that naturally occurring alterations in the expression levels of ICP would ultimately affect parasite virulence. In $T$. brucei rhodesiense, parasite CPs have been implicated in the traversal of the blood brain barrier (BBB), in endothelial cell signaling and more recently, in the regulation of endothelial cell gene expression $[37,38]$. In this sense, the levels of ICP may additionally influence this aspect of the parasite interaction with its mammalian host, ultimately controlling the participation of parasite CPs in BBB activation and in the pathology of the central nervous system during infection.

A different picture emerged when the role of ICP was investigated in Leishmania. Unlike chagasin in $T$. cruzi, in $L$. mexicana only a small proportion of ICP was found colocalizing with CPs in the endoplasmic reticulum and Golgi and the majority of ICP resides in vesicles that are apparently distinct from endosomes and the multivesicular tubule (MVT)-lysosome [9]. Furthermore, L. mexicana mutant lines lacking ICP grew normally in vitro, were as infective to macrophages as wild-type parasites, but had reduced infectivity to mice. These data led to the proposal that ICP has a role other than modulation of the activity of the parasite's own CPs and their normal trafficking to the MVT-lysosome via the flagellar pocket [9]. These findings indicate that natu- 
ral targets and the biological roles of ICPs might vary among trypanosomatids.

\section{Inhibitors of Serine Peptidases (ISPs)}

The investigation of the biological role of ISPs has been only recently initiated in $L$. major and little is known about their function in trypanosomatids [24]. The expression of these inhibitors is stage-regulated: ISP2 is found in three life cycle stages of $L$. major, being higher in amastigotes and metacyclic promastigotes, while ISP1 was detected only in promastigotes and metacyclic promastigotes. As previously mentioned, the expression of ISP3 was not detected, suggesting that it might be a pseudo-gene or that its expression is tightly regulated and initiated at specific points of the parasite's life cycle or during interaction with the host(s). In $T$. cruzi, we detected ISP2 in the three life stages, being higher in epimastigotes (Abreu MF, Reis FCG, Mottram JC, Lima APCA, unpublished). Although the sub-cellular distribution of ISPs remains unclear, they are most likely intracellular and no secretion of ISPs has been detected so far. Although ISPs seem to reside intracellularly, they could be released from dying parasites during infection, then becoming available to act on host proteins, as discussed below.

Host serine peptidases (SPs) are the probable targets of ISPs, and candidate SPs include chymotrypsin-like enzymes in the gut of the insect vectors [39, 40], or a variety of mammalian SPs involved in inflammation [41]. Postulated targets include SPs expressed by cells of the innate immune system (i.e., neutrophils, mast cells, macrophages), such as neutrophil elastase (NE), proteinase 3 , tryptase and cathepsin $\mathrm{G}$ (CG), as well as enzymes participating in the coagulation cascades, such as factor Xa. Recombinant ISP2 of T. cruzi or Leishmania inactivate $\mathrm{NE}$ and $\mathrm{CG}$ with moderate affinity ( $K_{\mathrm{i}} \mathrm{S}$ in the nanomolar range), having the potential to influence the outcome of the innate immune response during parasite infection. The premise that ISPs target host peptidases was first evaluated by analyzing the interaction between L. major mutant lines lacking ISP2 and ISP3 and macrophages [24]. We found that ISP-deficient parasites are phagocytosed by mouse macrophages more efficiently than wild type, via engagement of the complement type-3 receptor (CR3). Interestingly, the greater parasite uptake was due to the upregulation of the phagocytic activity of macrophages, regardless the nature of the internalized particle. Since the expression of ISP3 is apparently missing from wild type Leishmania, the phenotypes displayed by the mutants are most likely associated to the loss of ISP2 function. Importantly, the upregulation of phagocytosis and consequently, higher parasite infection, was strictly dependent on host cell SP activity, being fully reversed by inhibitors to S1A enzymes [24]. The involvement of S1A peptidases in macrophage phagocytosis or in Leishmania infection has not been elucidated, raising important questions on how ISPs may have evolved to control crucial steps of the host-parasite interaction. Further, our studies with T. cruzi lines overexpressing ISP2 also point to a possible role of this inhibitor in the infection of a variety of cell types (Abreu, MF, Reis, FCG, Mottram, JC, Lima, AP, unpublished), suggesting a similar function for ISPs in different trypanosomatids.

Intriguingly, the intracellular ISP2:3-deficient L. major were partially eliminated within $24 \mathrm{~h}$ of infection, and the remaining parasites slowly recovered growth after $48 \mathrm{~h}$, suggesting that ISP2 is required for the efficient establishment of infection in macrophages [24]. We found that the activity of host SPs triggered during parasite contact is responsible for the subsequent defect in the intracellular development and proposed that ISPs play an important role in inactivating host SPs at the initial stages of infection in order to guarantee successful survival of the parasite. The potential role of ISPs in modulating the interaction of Leishmania and neutrophils is currently under investigation, as well as the identification of the main enzymes which are regulated by ISPs during infection. This shall provided valuable information on how natural serine peptidase inhibitors produced by trypanosomatids contribute to their adaptation for survival in their hosts.

\section{ACKNOWLEDGEMENTS}

We are indebted to Flavia C. G. Reis for her contribution over the years. We thank M. Pilar Aoki for disclosing results prior to publication. We dedicate this review to Julio Scharfstein, whose vision and enthusiasm led to the discovery of chagasin. These studies were funded by CNPq, FAPERJ and the Wellcome Trust (081877).

ABBREVIATIONS
$\mathrm{CP}=$ Cysteine peptidases
$\mathrm{ICP}=$ Inhibitor of cysteine peptidases
$\mathrm{ISP}=$ Inhibitor of Serine Peptidases
$\mathrm{VSG}=$ Variant Surface Glycoprotein
$\mathrm{BSF}=$ T. brucei bloodstream form
$\mathrm{BBB}=$ Blood brain barrier
$\mathrm{MVT}=$ Multivesicular tubule
$\mathrm{NE}=$ Neutrophil elastase
$\mathrm{CG}=$ Cathepsin G
$\mathrm{CR} 3=$ Complement type-3 receptor

\section{REFERENCES}

[1] Rawlings ND, Tolle DP, Barrett AJ. Evolutionary families of peptidase inhibitors. Biochem J 2004; 378: 705-16.

[2] Turk V, Stoka, V, Turk, D. Cystatins: Biochemical and structural properties, and medical relevance. Front Biosci 2008; 13: 5406-20.

[3] Sajid M, McKerrow JH. Cysteine proteases of parasitic organisms. Mol Biochem Parasitol 2002; 120: 1-21.

[4] Mottram JC, Coombs GH, Alexander J. Cysteine peptidases as virulence factors of Leishmania. Curr Opin Microbiol 2004; 7: 37581.

[5] Irvine JW, Coombs GH, North MJ. Cystatin-like cysteine proteinase inhibitors of parasitic protozoa. FEMS Microbiol Lett 1992; 75: 67-72.

[6] Monteiro ACS, Abrahamson M, Lima APCA, et al. Identification, characterization and localization of chagasin, a tight-binding cysteine protease inhibitor in Trypanosoma cruzi. J Cell Sci 2001; 114: 3933-42.

[7] Rigden DJ, Mosolov VV, Galperin MY. Sequence conservation in the chagasin family suggests a common trend in cysteine proteinase binding by unrelated protein inhibitors. Protein Sci 2002; 11: 197177.

[8] Sanderson SJ, Westrop, GD, Scharfstein J, et al. Functional conservation of a natural cysteine peptidase inhibitor in protozoan and bacterial pathogens. FEBS Lett 2003; 542: 12-16. 
[9] Besteiro S, Coombs GH, and Mottram JC. A potential role for ICP, a leishmanial inhibitor of cysteine peptidases, in the interaction between host and parasite. Mol Microbiol 2004; 54: 1224-36.

[10] Riekenberg S, Witjes B, Saric M, et al. Identification of EhICP1, a chagasin-like cysteine protease inhibitor of Entamoeba histolytica. FEBS Lett 2005; 579: 1573-78.

[11] Pandey KC, Singh N, Arastu-Kapur S, et al. Falstatin, a Cysteine Protease Inhibitor of Plasmodium falciparum, Facilitates Erythrocyte Invasion. Plos Pathog 2006; 11: 1031-41.

[12] Sato D, Nakada-Tsukui K, Okada M, et al. Two cysteine protease inhibitors, EhICP1 and 2, localized in distinct compartments, negatively regulate secretion in Entamoeba histolytica. FEBS Lett 2006; 580: 5306-12.

[13] Huang R, Que X, Hirata K, et al. The cathepsin L of Toxoplasma gondii (TgCPL) and its endogenous macromolecular inhibitor, toxostatin. Mol Biochem Parasitol. 2009; 164: 86-94.

[14] Salmon D, Aido-Machado R, Diehl A, et al. Solution structure and backbone dynamics of the Trypanosoma cruzi cysteine protease inhibitor chagasin. J Mol Biol 2006; 357: 1511-21.

[15] Smith BO, Picken NC, Westrop GD, et al. The structure of Leishmania mexicana ICP provides evidence for convergent evolution of cysteine peptidase inhibitors. J Biol Chem 2006; 281: 582128.

[16] Cazzulo JJ. Proteinases of Trypanosoma cruzi: potential targets for the chemotherapy of Changas disease. Curr Top Med Chem 2002; 2: 1261-71.

[17] Figueiredo-Silva AA, Vieira LC, Krieger MA, et al. Crystal structure of chagasin, the endogenous cysteine-protease inhibitor from Trypanosoma cruzi. J Struct Biol 2006; 157: 416-23.

[18] Wang SX, Pandey KC, Scharfstein J, et al. The structure of chagasin in complex with a cysteine protease clarifies the binding mode and evolution of an inhibitor family. Structure 2007; 15: 53543 .

[19] Ljunggren A, Redzynia I, Alvarez-Fernandez M, et al. Crystal structure of the parasite protease inhibitor chagasin in complex with a host target cysteine protease. J Mol Biol 2007; 371: 137-53.

[20] Reis FCG, Smith BO, Santos CC, et al. The role of conserved residues of chagasin in the inhibition of cysteine peptidases. FEBS Lett 2008; 582: 485-90.

[21] Redzynia I, Ljunggren A, Abrahamson M, et al. Displacement of the occluding loop by the parasite protein, chagasin, results in efficient inhibition of human cathepsin B. J Biol Chem 2008; 283: 22815-25.

[22] Redzynia I, Ljunggren A, Bujacz A, et al. Crystal structure of the parasite inhibitor chagasin complex with papain allows identification of structural requirements for broad reactivity and specificity determinants for target proteases. FEBS J 2009; 276: 793-06.

[23] Musil D, Zucic D, Engh RA, et al. The refined 2.15 A X-ray crystal structure of human liver cathepsin B: the structural basis for its specificity. EMBO J 1991; 10: 2321-30.

[24] Eschenlauer SCP, Faria MS, Morrison LS, et al. Influence of parasite encoded inhibitors of serine peptidases in early infection with Leishmania major. Cell Microbiol 2009; 11: 106-20.

[25] Chung, CH, Ives HE, Almeda S, et al. Purification from Escherichia coli of a periplasmic protein that is a potent inhibitor of pancreatic proteases. J Biol Chem 1983; 258: 11032-38.
[26] McGrath ME, Hines WM, Sakanari JÁ, et al. The sequence and reactive site of ecotin. A general inhibitor of pancreatic serine proteases from Escherichia coli. J Biol Chem 1991; 266: 6620-25.

[27] McGrath ME, Erpel T, Bystroff C, et al. Macromolecular chelation as an improved mechanism of protease inhibition: structure of the ecotin-trypsin complex. EMBO J 194; 13: 1502-07.

[28] Seong IS, Lee HR, Seol JH, et al. The P1 reactive site methionine residue of ecotin is not crucial for its specificity on target proteases. A potent inhibitor of pancreatic serine proteases from Escherichia coli. J Biol Chem 1994; 269: 21915-18.

[29] Shin DH, Song HK, Seong IS, et al. Crystal structure analyses of uncomplexed ecotin in two crystal forms: implications for its function and stability. Protein Sci 1996; 5: 2236-47.

[30] Yang SQ, Wang CI, Gillmor AS, et al. Ecotin: a serine protease inhibitor with two distinct and interacting binding sites. J Mol Biol 1998; 279: 945-57.

[31] Eggers CT, Murray IA, Delmar VA, et al. The periplasmic serine protease inhibitor ecotin protects bacteria against neutrophil elastase. Biochem J 2004; 379: 107-18.

[32] Santos CC, Sant'Anna C, Terres A, et al. Chagasin, the endogenous cysteine protease inhibitor of Trypanosoma cruzi, modulates parasite differentiation and invasion of mammalian cells. J Cell Sci 2005; 118: 901-15.

[33] Sant'Anna C, Nakayasu ES, Pereira MG, et al. Subcellular proteomics of Trypanosoma cruzi reservosomes. Proteomics 2009; 9 : 1782-94.

[34] Cunha-e-Silva N, Sant'Anna C, Pereira MG, et al. Reservosomes: multipurpose organelles? Parasitol Res 2006; 99: 325-327.

[35] Aoki MP, Guiñazú NL, Pellegrini AV, et al. Cruzipain, a major Trypanosoma cruzi antigen, promotes arginase-2 expression and survival of neonatal mouse cardiomyocytes. Am J Physiol Cell Physiol. 2004; 286: C206-12.

[36] Santos CC, Coombs GH, Lima APCA, et al. Role of the Trypanosoma brucei natural cysteine peptidase inhibitor ICP in differentiation and virulence. Mol Microbiol 2007; 66: 991-02.

[37] Nikolskaia OV, Lima APCA, Kim YV, et al. Blood-brain barrier traversal by African trypanosomes requires calcium signaling in duced by parasite cysteine protease. J Clin Invest 2006; 116: 273947.

[38] Grab DJ, Garcia-Garcia JC, Nikolskaia OV, et al. Protease activated receptor signaling is required for african trypanosome traversal of human brain microvascular endothelial cells. PLoS Negl Trop Dis. 2009; 3: e479.

[39] Ramalho-Ortigao JM, Kamhawi S, Rowton ED, et al. Cloning and characterization of trypsin- and chymotrypsin-like proteases from the midgut of the sand fly vector Phlebotomus papatasi. Insect Biochem Mol Biol 2003; 33: 163-71.

[40] Yan J, Cheng Q, Li CB, et al. Molecular characterization of two serine proteases expressed in gut tissue of the African trypanosome vector, Glossina morsitans morsitans. Insect Mol Biol 2001; 10: 47-56.

[41] Pham CTN. Neutrophil serine proteases: specific regulators of inflammation. Nat Rev Immunol 2006; 6: 541-50.

[42] Rennernberg A, Lehmann C, Heitmann A, et al. Exoerythrocytic Plasmodium parasites secrete a cysteine protease inhibitor involved in sporozoite invasion and capable of blocking cell death of host hepatocytes. Plos Pathog 2010; 6: e1000825.

(C) Lima and Mottram; Licensee Bentham Open.

This is an open access article licensed under the terms of the Creative Commons Attribution Non-Commercial License (http://creativecommons.org/licenses/by-nc/3.0/) which permits unrestricted, non-commercial use, distribution and reproduction in any medium, provided the work is properly cited. 